\title{
Philosophy of Information: Revolution in Philosophy ${ }^{+}$
}

\author{
Joseph E. Brenner \\ International Center for the Philosophy of Information, A-1220 Vienna, Austria; joe.brenner@bluewin.ch \\ † Presented at the IS4SI 2017 Summit DIGITALISATION FOR A SUSTAINABLE SOCIETY, Gothenburg, \\ Sweden, 12-16 June 2017.
}

Published: 8 June 1017

\begin{abstract}
This paper for presentation at the 4th International Conference on the Philosophy of Information is based on what $\mathrm{Wu}$ Kun has described as the convergence of science and philosophy taking place under the impact of information science and philosophy. I address the question of the extent to which this trend may be considered a revolution in philosophy and whether it is it important and useful to so designate it. This is a preview of a joint paper by Professor $\mathrm{Wu}$ and the writer which will go more extensively into the nature of revolution and the philosophy of science as such.
\end{abstract}

Keywords: convergence; information; logic; philosophy; revolution; science

\section{Introduction}

\subsection{Information and Its Logic}

Under the leadership of Professor Wu Kun, studies of the characteristics of information from a philosophical as well as scientific standpoint are providing new insights into its nature and functionality. At the 2nd International Conference on the Philosophy of Information in Vienna two years ago, the focus of his work was on the convergence of the science and philosophy of information toward a new Science-Philosophy of Information. I explore here the question of whether the movement in philosophy which Wu has identified constitutes, or not, a revolution in philosophy and, if yes, what the consequences of such a revolution might be.

The approach of $\mathrm{Wu}$ and the writer to information defines it as a complex process and focuses its role in other complex real processes such as mind, personal identity, life, and change in general. Underlying and supporting such approaches, I have proposed a non-standard logic which I have called Logic in Reality (LIR). As I will try to show, LIR is particularly suited to the formalization and explication of the antagonistic, interactive ontological and epistemological relations involved in information and other complex processes. These include revolutionary as well as evolutionary phenomena, and I will accordingly start with a summary of the work of Thomas Kuhn as a model for this study.

\subsection{The Kuhnian Model}

The canonical model for this research on the revolutionary aspects of information is the now classical 1962 study by Thomas Kuhn: The Structure of Scientific Revolutions. It is immediately clear from subsequent work by Kuhn and others, both his followers and detractors, that there is no consensus either on what criteria define a revolution in science or whether the changes in progress in science and society constitute one or more revolutions. In this paper, I will address two separate but obviously related questions: (1) is it correct to say that science and philosophy are converging in some manner and (2) is it important and useful to call attention to this development? Judging from 
the enormous amount of discussion of Kuhn's work that has taken and is still taking place, it would seem that whether or not a set of concepts constitute a revolution or not is a subject of great academic interest, if nothing else.

This study has, however, identified an essential way in which a revolution in philosophy differs conceptually from a revolution in science: the latter refers to the dynamic form of changes in scientific theory; the former points to discontinuities in world-views. Science has a role, but there is a 4th logical and ontological dimension of incommensurability as a marker for revolution in addition to the epistemological dimensions identified by Kuhn. It is this logical dimension that it would now seem desirable to explore.

\subsection{Outline of Paper}

In Section 2, then, I summarize the relevant principles of Logic in Reality and compare them with some alternates. Section 3 presents an approach to a theory of information and points to the existence of a current 'informational turn'. Section 4, logically, suggests the impact the informational turn is having on philosophy. In Section 5, I revisit the recent work done by Wu and myself on the convergence of science and philosophy that is a consequence of this impact. Section 6 proposes that the changes taking place can in fact be considered as a revolution, with a degree of discontinuity that is sufficient even if not absolute. I conclude in Section 7 with the suggestion that a revolutionary attitude toward philosophy would be the most appropriate one.

\section{Logic and Logic in Reality (LIR)}

The logical system I have designated as Logic in Reality (LIR) applies to the fields mentioned to which I feel standard binary, truth-functional logic does not. It was first proposed by the Franco-Romanian thinker, Stéphane Lupasco (Bucharest, 1900-Paris, 1988). This scientific-philosophical perspective is essentially a process view of nature and change. I define two kinds of thermodynamic change, linear and interactive where linear refers to single-element energetic processes in a simple gradient, like falling water. Interactive change describes the evolution of complex processes whose elements are mutually determining. They are related such that the actualization of one implies the potentialization of the other, alternately and reciprocally, without either ever going to the ideal limits of 0 or 1, except in trivially simple cases. One can list the movements of opposing elements of the process as follows:

- Movement from actuality to potentiality and vice versa

- Movement from identity (unity, homogeneity) to diversity (multiplicity, heterogeneity) and vice versa

- Emergence of new entities from the mid-point where the degree of actuality and potentiality is the same.

These rules operate at all levels of reality and define a logic, which I have called Logic in Reality (LIR), allowing inferences about the further evolution of the processes in question. It should be emphasized that LIR is a logic of relations and interactions of the elements of real processes, not those of propositional logic or mathematical logic. Many of you may see, rather, a relation of the Lupasco system to ancient Chinese ideas about natural science, as implied in the Tao Te Ching on the one hand, and social science-the individual and the state-exemplified by the Analects of Confucius. I indicate just a few further aspects of Logic in Reality relevant to today's discussion:

- LIR discusses philosophical problems in physical, dynamical terms that do not require abstract categorial structures that separate aspects of reality.

- The critical categorial feature of the LIR process ontology is the non-separability of opposing phenomena, e.g., two theories or elements of phenomena, e.g., syntax and semantics, types and tokens, at biological and cognitive levels of reality. 
- The philosophy of LIR can be very rapidly characterized as a non-naïve dualistic realism that assumes a real, interactive relation between all the classic dualities when they are instantiated in reality.

Some examples of these dualities are the following:

- Quantum Level: Uncertainty Principle

- Biological Level: Antibody/Antigen Interactions

- Cognitive Level: Conscious/Unconscious

- Sociological Level: Left -Right Swings

- Cosmological Level (?): Dark (Negative) Energy/Ordinary Matter-Energy

To conclude this introductory Section on logic, As I stated at the 2010 Foundations of Information Science Conference in Beijing, the Universal Logics of Professors He Huacan and Jean-Yves Béziau are the most complete descriptions available of the mathematical dialectic aspects of propositional logic. They therefore include and systematize paraconsistent and paracomplete (intuitionist) logics as well as so-called fuzzy logics. To the extent that vagueness is a rigorous property of real systems, fuzzy universal logic may be considered as an approach to reality compared to classical binary logic. However, it is limited due to its retention of propositions or statements, or their mathematical equivalents, as its elements. In contrast, Logic in Reality describes the non-mathematical dialectical aspects of existence. Both may be needed for the science of both information and of other complex cognitive processes such as memory-forgetting, personal identity and intelligence. My hope is that the approach of Logic in Reality to intelligence, in which the non-mathematical properties of intelligence as well as information emerge clearly, may favor a proper balance between the two approaches of propositions vs. process, as well as the more complex normative worldview embedded in the latter.

\section{The Dualisms of LIR}

In this logical approach to reality, the opposing elements in a phenomenon are not sentences, but may be epistemological or ontological entities: molecules, theories or people, individuals or groups. I have listed some of the pairs of opposites most critical for this discussion, with emphasis on the process nature of these logical elements.

- Knowledge (Knowing) Ignorance (not-Knowing)

- Knowledge_as_Such Intuition

- Philosophy (Philosophization) Science (Scientification)

- Information_as_Energy Information_as_Meaning

- Memory Forgetting

- Presence Absence

- Sharp Fuzzy

- Figure Ground

Note my progressive inclusion of characteristics of pictorial art, which are at the same time those of information, especially in the view of Terrence Deacon. They also apply directly to the understanding of classical Chinese painting. The philosophical view of these elements of knowledge as information or informational in the Philosophy of Information as a Metaphilosophy, developed by Professor Wu Kun, rejoins the more scientific LIR view of these elements as energetic processes.

\section{Information and Informational Turn}

Information is an entity or process, or set of entities and processes, that is unique in both science and philosophy. It requires acceptance as a concept that cannot be defined as an identity, but only as a dynamic interactive dualism of matter-energy (ontological properties) and meaning (epistemological properties). Cognitive processes, as well as their corresponding analyses and theories, instantiate similar dualities, of which the prime example is that of self and other. 
Information is somehow associated with or constitutive of existence, but it has proven notoriously difficult to define and characterize, due to its multiple duality: it has both physical and apparently non-physical components, both a real dynamic and algorithmic descriptions. I note that both science and philosophy involve the observation of regularities in nature which only differ in the degree of certainty to which can be ascribed to them. Greater rigor in philosophy does not come easily; however, the properties of information common to both science and philosophy can be used to reconcile the physical, scientific properties of information with its epistemological and philosophical characteristics as a carrier of meaning. Both a physics (science) and mutually consistent philosophy of information are required and that both the philosophy and science of information must inform one another.

\section{Wu Kun and the Informational Turn}

$\mathrm{Wu}$ Kun has clearly brought out the ontological impact of information on philosophy. In his recent papers, $\mathrm{Wu}$ Kun presents detailed arguments for a new perspective on philosophy and science and the changes which they are undergoing under the influence of the informational activities of the society. I indicate five position statements that constitute the 'backbone' of Wu's metaphilosophical theory:

- Mind-Matter Dichotomy

With some notable exceptions, the bulk of philosophical doctrine is still based on the categorial separability of mind and matter. Despite advances in neurobiology and related sciences, the laws of reasoning and logic remain isolated from physical science, a part of semiotics as discussed above. $\mathrm{Wu}$ 's first contribution to the discussion is to show that the existence of information requires a resegmentation of the existential field, making the mind-matter dichotomy on which much current thinking is still based untenable.

- The Science-Philosophy Dichotomy

The concomitant acceptance of the philosophical duality of information and the recognition of its physical duality abrogate any absolute separation between science and philosophy. This principle, within the Philosophy of Information (PI), is becoming applicable to science and consequently the Philosophy of Science (PS), as well as philosophy itself.

- The Position of Information in Reality. Properties

Any complex real entity, e.g., a person, can be considered as constituted by the totality of the informational processes, past, present and potential in which he is involved. The intermediate stages which bridge the gap between external and internal reality are all informational. This approach is consistent with Deacon's approach to the hierarchy of dynamics and second-order constraints necessary for the emergence of life.

- Implications for the Information Society

$\mathrm{Wu}$ sees the multi-dimensional informational structures and processes in the society as reducing domination by central governmental control. They thus support an increase in principle in democracy, including information as a commons. Further work is needed, however, to determine if there is a direct correlation between the operative principles of Wu's Philosophy of Information and the political change necessary to implement them.

- The Informational Turn

As a discipline, Information Science has a unity by virtue of its spanning human knowledge from philosophy to science and engineering, with both vertical and horizontal relations between its component sub-disciplines. The further integration of Information Science and the Philosophy of Information implies a major Informational Turn in the current practice of both science and philosophy. I will return to this in the next Section on the Convergence of Information Science and 
Philosophy, a concept first proposed in its current form by $\mathrm{Wu}$ and in Section 5 on its revolutionary aspects as such.

\section{The Impact of the Informational Turn on Philosophy}

Philosophical changes can occur in different levels of philosophy: the effect, significance and value of these philosophical changes for the development of philosophy are different. In Wu Kun's conception, the informational turn of philosophy was different from other philosophical turns, due to its position at the level of the highest paradigm of philosophy which in this view is the mode of domains of existence. Generally speaking, only philosophical changes at the level of ontology are meaningful and valuable. According to $\mathrm{Wu}$ Kun, different schools of philosophy of information have had different interpretations for or ways of regarding of the role of information in ontology: (1) as an objective reality embedded in matter; (2) as one of the forms of mind (spirit), to be eliminated from the objective world; (3) as something different from both matter and mind. The first two interpretations have not changed the traditional mode of division of realms of existence, but the third has. This change resulted in the fundamental change in philosophy that can be called a "brand new philosophical revolution".

According to $\mathrm{Wu} \mathrm{Kun}$, there were major limitations in the appreciation of the nature of the philosophy of information in science and philosophy both in China and elsewhere. Most scholars comprehended the nature of the philosophy of information only at the level of philosophical problems in information science and information technology. According to this view, philosophy of information can only be a domain philosophy like the philosophy of physics, etc. Because these approaches are attached to the existing relevant specific philosophical theories or scientific and technological theories, they are consciously or unconsciously tied to the narrowness and limitations of these original theories and these approaches do not well reveal the unique significance and value of informational approaches to problems. The information theory based on these approaches does not belong to Information Science at its highest level nor does it have the nature of a General Philosophy of information or a Unified Information Science.

A further key principle in Wu's theory is his 'dual critique' of philosophy and science. He calls research approaches in above two directions "the analogy of philosophy": to draw an analogy with circumscribed theories and views of specific science and stale theories and views of existing philosophy. This analogy reflects the vulgar, conservative and rigid conception of philosophy. He advocates that the standpoint of "philosophical critique" must be applied to the study of information problems. The objective is to achieve a dual critique and dual transcendence in knowledge: to criticize and transcend circumscribed theories and views of specific science and stale theories and views of existing philosophy. This leads to a dual revolution: a revolution in a world-view of reality and of philosophy itself $(\mathrm{Wu}, \mathrm{K}$. Philosophical Forced Analogy and Philosophical Critique. Social Sciences in China 1995, (4), 117-125).

\section{Aspects of the Convergence of Information Science and Philosophy}

The most general theories and methodologies of Information Science are becoming the new normal form of scientific research/behavior, and this new form is clearly penetrating and influencing all knowledge. When the relevant information scientific principles and methods are applied to existing traditional disciplines, especially, the conceptual aspects of the biological, cognitive and social sciences, they result in a transformation that gives new meanings to them. Following $\mathrm{Wu}$, I thus first discuss the nature and impact of the Philosophy of Information and Information Science from a metatheoretical standpoint, as scientific phenomena in their own right. To help decide whether or not this state of affairs constitutes a revolution in philosophy as well as science is the objective of this inquiry.

The transformation of philosophy by science can not be achieved automatically by counting on scientific successes in themselves, but depends on relevant critiques of science by philosophy. In the West, the concept of a General Theory of Information (GTI) was first stated explicitly by Mark 
Burgin in 2003, later expanded and developed in a major book in 2010. The concept of a Unified Theory of Information has been developed most completely by Wolfgang Hofkirchner.

In China, $\mathrm{Wu}$ Kun has suggested that a Unified Science of Information (USI) would be a disciplinary system including all human knowledge from philosophy to science to technology. Such a Unified Science of Information would favor further convergent integration of science and philosophy. Thus, the Philosophy of Information could be viewed as a part of a general Science of Information, and the Science of Information as achieving its real unity by its foundation in the general principles of philosophy. In this vision, the Unified Science of Information is the scientific basis of a general Philosophy of Information, and the Philosophy of Information is the general theoretical precondition of a unified and unifying Science of Information. In Wu's opinion, the establishment of a Unified Science of Information and the mature development of a Philosophy of Information should be a process of convergence, two sides of a new and integral developing pattern of contemporary human knowledge.

This convergence, toward a Unified Science-Philosophy of Information (USPI), is a current theme of Professor Wu's work. His most recent work points to the convergence of science and philosophy under the impact of the revolution in Information Science. In his view, a true Philosophy of Science, which should obviously be grounded in science, thus cannot be separate from and supervene on science. The current dependence of philosophical development on scientific development indicates that science is the strongest and most basic driving force for the transformation of philosophy.

The principles of Logic in Reality apply again here: in such a USPI, the individual components do not lose their identity, but remain in a active dialectic relation to one another. One's mind alternates between a predominantly philosophical and predominantly scientific perspective.

But is this true? Is it not more correct to say that science and philosophy are converging and diverging, becoming similar and dissimilar at the same time? From my experience, I know that organic chemistry is becoming more are more difficult and complex, requiring expertise in computer science and technology that was not necessary when I was in school. Science in this sense is becoming less and less accessible to non-scientists-philosophers among them. At the same time, the general principles of life and mind and aspects of the evolution of certain biological processes are being taken into account in a new and transdisciplinary way, for example in the Integral Biomathics of Plamen Simeonov and his associates.

\section{Revolution and Revolution in Philosophy}

As noted at the start of this paper, the concept of one or several revolutions in science has been accepted as a valid topic of research for some time. However, if one looks today at current volume of a major Western philosophical journal such as SYNTHESE, it is difficult to find references to any specific role of information, let alone a revolutionary one, even in articles dealing with foundational issues of ontology and quantum mechanics.

It thus has been a major objective of this article and of our joint work is to begin to redress this situation by documenting the way in which information can have a major impact on philosophy, on science and hence on the philosophy of science itself. As noted at the start of this paper, the concept of one or several revolutions in science has been accepted as a valid topic of research for some time. One notes of course the work of Thomas Kuhn and his commentators. The concept of a revolution in philosophy has also previously been formalized by Marx and in the 1950 by Ayer and others, but it is certainly not the subject of extensive current debate. However, from the point of view of Wu Kun, a new 'quiet' revolution in philosophy has been taking place in China since the early 1980's, with the recognition of the major impact on philosophy of the philosophy of information, considered as a metaphilosophy. This revolution is expressed in radical changes to the way fundamental philosophical problems can be conceived of and discussed. Wu has stated that in fact a revolution is occurring from the standpoint of the Philosophy of Information although the number of individuals involved, judged from an rough survey of publications, is probably still very limited. 
The hermeneutic link that I establish is the following: information requires the re-introduction into science as well as philosophy of the neglected (because poorly grounded) dialectics of subject and object. To the extent that some non-Chinese thinkers embody and support a subject-object dialectics in their work, they participate ipso facto in the revolution in philosophy. The underlying humor in this situation is not a basis for not giving it the serious attention it deserves. The establishment and development of information science and technology occupy a unique and critical position in the 20th Century science, constituting in the views of some workers a scientific and technological revolution. In 1999, Fang listed no less than six types of revolution in this field. Most recently, in the phrase of Floridi, information is the $4^{\text {th }}$ major revolution in the series communication (Gutenberg)-industrial-scientific. We are witnessing the establishment of an economy of knowledge and information and the emergence of a knowledge-based information society. In parallel, over the last 40 years, philosophers have begun to develop a Philosophy of Information as a field in its own right, in order to bring some conceptual order into the wide variety of conceptions of information.

This paper focuses on some of the theoretical aspects of the current revolution in philosophy which $\mathrm{Wu}$ and I have identified. As he points out, the application of the Philosophy of Information involves a fundamental theoretical transformation operating the level of the highest paradigm of philosophy, that which discusses the structure of existence. It is this fundamental theoretical transformation which constitutes the revolution of philosophy. Its consequences can be expected to be further changes in all basic and non-basic problems and fields of philosophy. In the companion paper on the revolution in philosophy which $\mathrm{Wu}$ Kun has prepared for this Conference, he emphasizes some specific areas in which changes in ideas are taking place.

\subsection{The Logic of Change and Revolution}

Change is ubiquitous in nature, and change in living systems, especially human thought and behavior is the most complex form of which we know. For human beings, two terms characterize change as rate, slow evolution and rapid revolution. The first applies to the biological or cognitive realm, the second only to cognitive disciplines and social structures. These processes can be further characterized by (1) their relation, that is, whether there is a continuous spectrum of change between them, or whether revolution implies a discontinuity, and (2) generality, the number of individuals involved and their degree of consciousness of being involved in drastic change. I claim that a change in the domain of philosophy has occurred and is occurring that, although not instantaneous nor widespread, is sufficiently drastic to be called a revolution. Logic in Reality is a logic of change par excellence, and it would seem to provide both a framework and a language for the discussion of the revolutionary changes in philosophy.

\subsection{In Relation to Information Science}

As noted above, Wu Kun has characterized his Philosophy of Information as a Metaphilosophy since it discusses ways in which all theories are constituted and operate in the evolving informational world. Following his outline for information, one may construct an 'informational' model of knowledge which includes as its proper parts Information Science and its data. In the framework of Logic in Reality, the essential point is to recognize what brings together, rather than what differentiates or separates, the epistemological and ontological elements of information. In my LIR view, these are part of the content of Information Science.

There is an additional consequence of the proposed convergence occurring in thought. It is that if the philosophical implications of a scientific discovery are equally fundamental and revolutionary. In this case it becomes more and more difficult to give priority to science as opposed to its philosophy, although they remain distinct. In the Copernican revolution, man was forced to see himself as being no longer at the center of a solar universe. Later, our importance was reduced further by the understanding that our solar system is a tiny spot at the edge of average galaxy, of which there are billions. Today, we must absorb and integrate into our thinking and being that the form of 'ordinary' matter-energy of which we are composed is again only a small fraction of the 
total of which the universe is composed and evolves. I have suggested elsewhere that a duality of mutual transformation exists between ordinary matter-energy and negative energy. (The problem of the relation of dark matter to ordinary matter is totally open at this time, since there do not appear to be any interactions between them that could justify applying logical rules which refer to interactions). A 'logic of duality' enables one to be more comfortable about living with the ignorance that accompanies, dialectically, such increases in knowledge. They must be accompanied by increases in humility, or one becomes lost in Promethean technological fantasies.

\subsection{In Relation to Science in General}

Nothing in my discussion so far requires that any revolutionary movement be absolute, and one therefore must be able to distinguish degrees of revolutionarity. The criticism to be made here is that there will be two sets of observers whose positions must be taken into account: those who consider that a theory is moderately revolutionary, despite the absence of major discontinuity. Such a position cannot be distinguished from some forms of evolution. In fact, the absence of total separation between revolution and evolution is a second-order dialectical opposition to which the principles of LIR apply. Another process, to which attention is being paid in the field of biology, is devolution, a movement backwards toward prior states, either more or less complex. Revolution and devolution thus constitute another duality in the same sense.

It is important to note that there are others fields in which a convergence of science and philosophy has taken place without being considered revolutionary. The sciences in question are biology and neuroscience, without prejudice to their specificities of methods or theories (SEP reference).

\section{Conclusions: The Revolutionary Attitude}

$\mathrm{Wu}$ Kun has defined the Informational Attitude or Stance (IS) as a philosophical position most appropriate for, and not separated nor isolated from, the emerging science and philosophy of information itself. IS requires attention to the informational aspects of complex processes as a methodological necessity.

Application of an Informational Stance would involve defining, for each concept in information research, in all of science and in fact of all knowledge not only its scientific and philosophical aspects but their interaction and co-modification. The major consideration of such a stance associated with a Unified Science-Philosophy of Information is thus that of giving proper value to its qualitative, non-measurable and non-computable components. It should never be said that qualitative relations and inequalities which follow the rules of Logic in Reality are more important or necessary than the quantitative equalities, only that they are no less fundamental.

Together with Wu Kun, I consider that a Revolutionary Attitude is now a necessary part of the intellectual world, embodying several functions. It would be revolutionary, for example, for people to change the dynamics of their acceptance of the published literature, exercising the principle of tolerance but also rigor that Nicolescu has insisted are the main attributes of the Transdisciplinary Attitude. Other multi- and interdisciplinary movements, such as that of Translational Medicine, emphasize the need for a radical break with accepted ways of realizing the medical benefits to the patient of fundamental research in biology. The consequences of such a Revolutionary Attitude remain to be defined, but they hopefully would accompany a corresponding positive change in the economic and social structure of society as a whole.

Conflicts of Interest: The authors declare no conflict of interest.

(C) 2017 by the author. Licensee MDPI, Basel, Switzerland. This article is an open access article distributed under the terms and conditions of the Creative Commons Attribution (CC BY) license (http://creativecommons.org/licenses/by/4.0/) 